\title{
Características epidemiológicas de los fallecidos durante el terremoto y maremoto de Chile 2010
}

\author{
JORGE LASTRA T. ${ }^{1}$, GUILLERMO GUZMÁN C. ${ }^{2}$, CONSUELO CONEJEROS R. ${ }^{1}$, \\ GUILLERMO SUÁREZ V. ${ }^{1, a}$, ORLANDO CHÁVEZ F. ${ }^{1, a}$
}

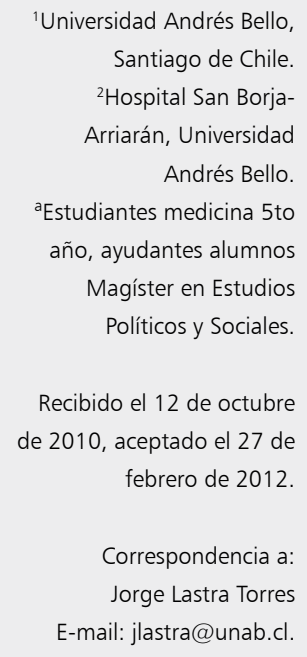

'Universidad Andrés Bello,

Santiago de Chile.

${ }^{2}$ Hospital San Borja-

Arriarán, Universidad

Andrés Bello.

aEstudiantes medicina 5to

año, ayudantes alumnos

Magíster en Estudios

Políticos y Sociales.

Recibido el 12 de octubre de 2010, aceptado el 27 de febrero de 2012.

Correspondencia a: Jorge Lastra Torres

E-mail: jlastra@unab.cl.

\section{Epidemiological profile of deaths after the 2010 earthquake in Chile}

Background: On February 27, 2010 a powerful earthquake followed by a tsunami stroke Chile. The study of mortality during this emergency can provide important public health information. Aim: To describe the main characteristics of people who died during the earthquake and the following three months. Material and Methods: Cross sectional analysis of death records databases obtained from Department of Health Statistics and Information of the Ministry of Health and the Coroner office. Results: Until May 25, 2010, 505 corpses were completely identified. Seventy two of these corresponded to people aged 80 years or more. The higher age adjusted death rates per 100,000 inhabitants were observed among subjects aged more than 80 years and those aged 70 to 79 years (22.6 and 7.7 respectively). The higher rates of deaths were observed in regions where the earthquake had a higher intensity and coastal regions affected by the tsunami. The causes of death were trauma in $75 \%$ of cases and drowning in 25\%. There was no association between the Mercalli scale of earthquake intensity and rates of death. Among deceased subjects, there was a concentration of unemployed, under educated and low socioeconomic status subjects. Conclusions: After the earthquake, the higher rates of deaths occurred among older people and in the region of the epicenter of the earthquake. Most deaths were due to trauma.

(Rev Med Chile 2012; 140: 732-739).

Key words: Disasters; Fatal outcome; Mortality; Public Health; Socioeconomic factors.
E 1 día 27 de febrero del año 2010 se produjo en Chile un terremoto de 8,8 grados Richter $y$ un maremoto, que afectaron a su territorio entre las regiones $\mathrm{V}$ y $\mathrm{X}$ en una extensión aproximada de $800 \mathrm{Km}$ de longitud; con una cifra de fallecidos identificados al 25 de mayo de 2010 de 505 a los que sumaron 10 posteriormente. Otros 25 cuerpos permanecen hasta hoy desaparecidos $\mathrm{y}$ se atribuyen al mismo desastre.

Tanto por la importancia cuantitativa como por la significación que el fenómeno ha tenido, se justifica conocer y analizar cuál es el perfil epide- miológico de los casos identificados, evaluando diversas variables del registro de identificación y su respectivo certificado de defunción, y comparando con los patrones de muerte en cataclismos similares en otras partes del mundo.

En Chile, los desastres de este tipo son frecuentes, desde los primeros registros de la época de colonización ya existen antecedentes de ellos como una parte permanente de su historia; muchos de ellos ocupando los primeros lugares de intensidad en el mundo. Por lo mismo, los cambios en materia de obras civiles muestran un progreso 
continuo, tanto que un sismo de esta naturaleza en cualquiera de las principales capitales del resto de los países, latinoamericanos, norteamericanos o europeos provocaría un desastre de proporciones incalculables, como por ejemplo ocurrió en Puerto Príncipe donde un sismo de 7 grados de intensidad Richter, causó casi trescientos mil muertos conocidos y tres y medio millones de damnificados. Sin embargo, tanto en nuestro país como en el mundo el análisis de las víctimas ha sido escasamente estudiado, difundido y considerado a la hora de diseñar políticas de salud, destinadas a proteger los grupos de mayor riesgo. Por esta razón, conocer los efectos de este tipo de desastres en materia de salud pública y cuales son los grupos que se ven mayormente afectados es muy importante.

Los efectos de este tipo de desastres se pueden clasificar en inmediatos y mediatos:

a) Efectos inmediatos, corresponden a aquellos que se producen como resultado directo de la energía liberada por el terremoto y tsunami, y que causan muertos y lesionados.

b) Efectos mediatos, son los derivados de los daños a la infraestructura de salud que reduce la respuesta asistencial y al medio ambiente, que afectan la calidad de vida y ponen en riesgo a la población por enfermedades transmisibles, trastornos de salud mental derivados de la experiencia traumática, alteración de la nutrición por escasez de alimentos, movimientos migratorios de las poblaciones, emplazamientos masivos de albergados o campamentos precarios, el aumento de los discapacitados y, por último, los daños a la salud de los enfermos crónicos que enfrentan en estos desastres condiciones de complicación, no pocas veces con consecuencias fatales como lo describen otros estudios ${ }^{1}$.

Respecto de los afectados, la literatura es consistente en señalar que son fundamentalmente los pobres, los viejos, los desvalidos, discapacitados y menores de edad ${ }^{2-6}$.

El objetivo de este estudio es conocer las principales características epidemiológicas de los fallecidos durante el terremoto y el maremoto de Chile el 27 de febrero de 2010, con el propósito de evaluar si se reproduce el perfil observado en otros siniestros de este tipo y orientar la política pública para continuar reduciendo el impacto de fenómenos de este tipo en situaciones futuras.

\section{Material y Método}

Este es un estudio observacional, descriptivo, que da cuenta de las principales características epidemiológicas de los fallecidos a causa del sismo y tsunami del 27 de febrero en Chile del año 2010.

Los datos fueron extraídos de fuentes oficiales del Servicio de Registro Civil e Identificación, el Servicio Médico Legal e informados al Departamento de Estadísticas e Informaciones del Ministerio de Salud de Chile. Este registro de los datos, siguió los procedimientos habituales de identificación de fallecidos en Chile, por parte del Servicio de Registro Civil e Identificación, que verifica la identidad del cuerpo a través de pruebas dactiloscópicas; así como de parte del Servicio Médico legal, que realiza las pericias tanatológicas y que entrega el Certificado de Defunción. Finalmente, todos estos antecedentes son remitidos al Departamento de Estadísticas e Informaciones de Salud del Ministerio respectivo, que es quien dispone de la base de datos que hemos analizado y que nos la ha entregado a solicitud de la Universidad Andrés Bello para este estudio.

El proceso de registro de la información no fue fácil, por la suma de procedimientos y las dificultades de cada uno de ellos. El rescate de los cuerpos de entre los escombros y de la costa, la disponibilidad de identificación de las huellas dactilares y la capacidad de diagnóstico tanatológico fueron los principales responsables del prolongado proceso de recolección de información. Por esta razón, cuando se ha considerado recuperada la información de la mayor parte de los cuerpos, es que hemos decidido hacer este estudio y realizar esta publicación.

Esta base de datos fue analizada a través de estadísticas descriptivas; tabulados con distribución de frecuencias, en función de las principales categorías que se estudiaron: sexo, edad, residencia, ocupación, nivel de instrucción y diagnóstico de la causa de muerte principal. También se construyeron tasas de mortalidad que expresan la frecuencia de decesos por esta causa por 100.000 habitantes, según residencia y según edad, como una forma de dimensionar la probabilidad o riesgo de morir que tuvieron estas poblaciones afectadas por el evento según las variables comentadas. Adicionalmente, se realizó la prueba de $\chi^{2}$ como prueba de bondad de ajuste para determinar la relación de fallecidos 
según grupo etáreo, con la estimación de fallecidos que debió existir por grupo etáreo si el fenómeno hubiera afectado de manera homogénea a toda la población. Esta misma prueba estadística se utilizó para establecer la significación de la diferencia observada en los tipos de traumatismos como causa de muerte según sexo.

Finalmente, se aplicó el coeficiente de correlación como medida del grado de asociación entre las variables cuantitativas, tasas de muerte por región y la intensidad del terremoto según escala de Mercali.

La búsqueda bibliográfica realizada utilizó los buscadores clásicos de la literatura científica latinoamericana y de Estados Unidos de Norteamérica; "Scielo" y "Pubmed", así como los buscadores tradicionales de literatura gris.

\section{Resultados}

A la fecha del estudio, el 25 de mayo de 2010, se habían identificado 505 cuerpos. Posteriormente, el Servicio Médico Legal agregó 10 cuerpos, que no fueron incluidos en este estudio, como tampoco ocurrió con otros 25 cuerpos que quedaron registrados como desaparecidos por el tsunami, por el Informe de la Fiscalía Nacional que estudió la situación $(2011)^{7}$.
Los resultados se presentan de acuerdo con la distribución de fallecidos por sexo, edad, residencia, nivel de instrucción, ocupación y diagnósticos.

Los resultados de estos datos son los siguientes:

La Figura 1 muestra los fallecidos por sexo y se puede apreciar una distribución homogénea, similar a la nacional.

En cifras totales, el grupo de edades que presentó más fallecidos es el de 80 y más, con 72 casos, le siguen el grupo de 50 a 59 y de 60 a 69 con 70 y 67 casos respectivamente (Figura 2). Es decir, principalmente se vio afectado el grupo de los adultos mayores.

En las tasas de fallecidos por edad, que corresponden al número de fallecidos por 100.000 habitantes de cada uno de los tramos de edad considerados; el grupo que presentó una cifra mayor es el de 80 y más y le sigue el de 70 a 79 años con 22,6 y 7,7 por 100.000 habitantes

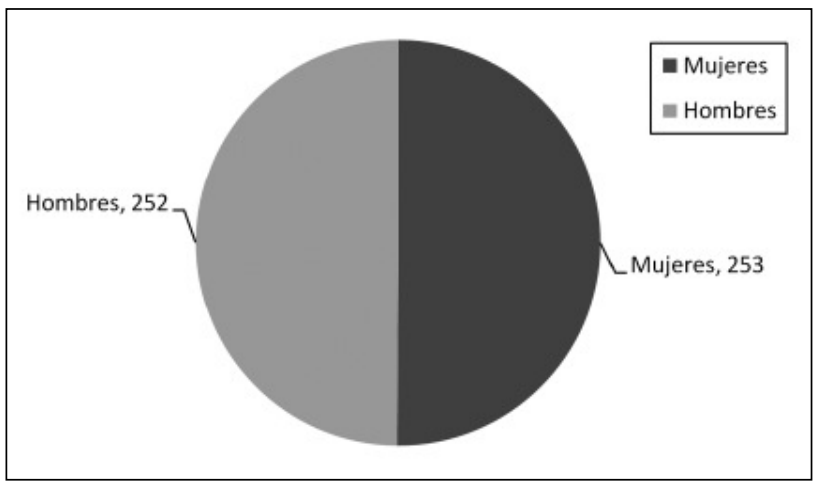

Figura 1. Número de fallecidos por sexo.

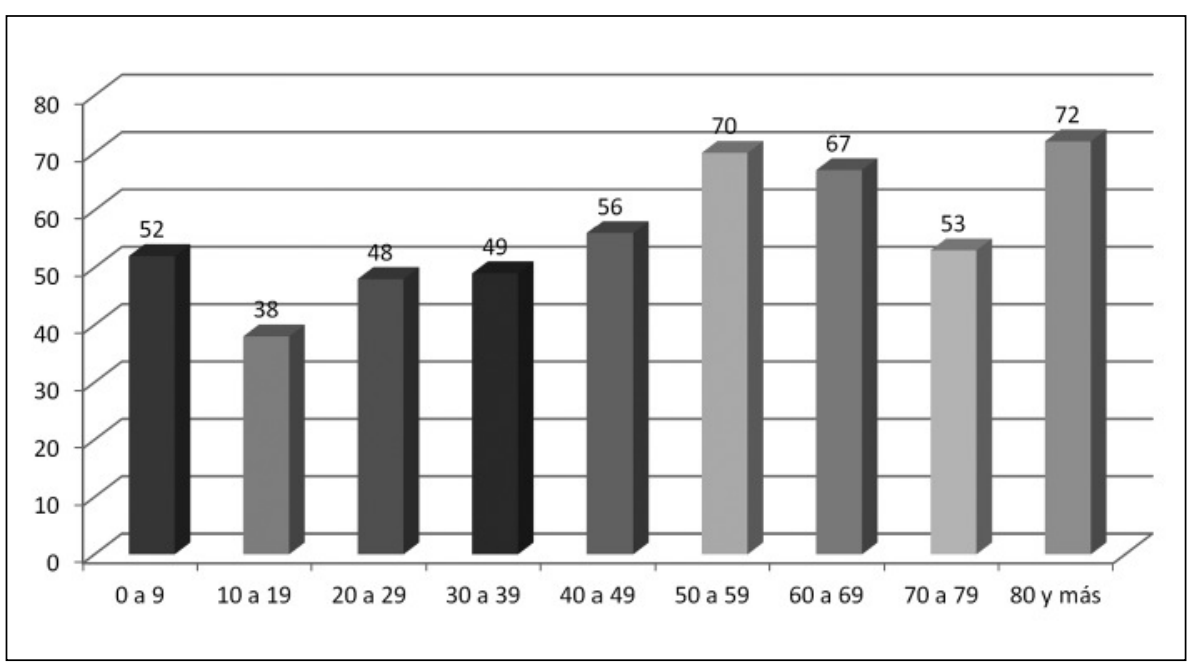

Figura 2. Número de fallecidos por rango de edad. 
respectivamente (Figura 3). Esta distribución no corresponde a la de la mortalidad que debiera presentarse como resultado de un fenómeno que afecta de manera homogénea a todos los habitantes, independiente de su edad. La prueba de $\chi^{2}$, da un resultado (549) que informa de una probabilidad menor que el nivel de significación, por lo tanto, se puede afirmar que los fallecidos por el terremoto según edad no sigue la distribución homogénea que debía esperarse.

Según residencia, el mayor número de fallecidos se observó en la Región del Maule con 243 y una tasa de 26,76 por 100.000 habitantes, superior a las tasas de Bío Bío, Libertador General Bernardo O'Higgins y Metropolitana (Figuras 4 y 5 ).

A nivel de provincias, Cauquenes y Talca en la Región del Maule, y Colchagua en la del Libertador, son las que presentaron las mayores tasas (Figura 6). Por su parte, las comunas con más casos fueron Constitución, Talca, Pelluhue y Cauquenes, todas de la Región del Maule, con 78, 27, 21 y 19 respectivamente (Figura 7).

Las tasas por regiones y comunas corresponden a los casos de fallecidos por 100.000 habitantes en cada uno de esos territorios.

La distribución de los fallecidos mayores de 18 años, según nivel de instrucción, muestra que el $64 \%$ tiene básica o menos (Figura 8).

Considerando la ocupación, 354 de los 505 eran inactivos y los ocupados estaban principalmente en categorías de obreros, empleados y trabajadores por cuenta propia (Figura 9).

Respecto de los diagnósticos básicos, el $75 \%$ de las muertes se produjeron a causa de traumatismos y sólo el $25 \%$ por ahogamiento o sumersión y en los tipos de trau-

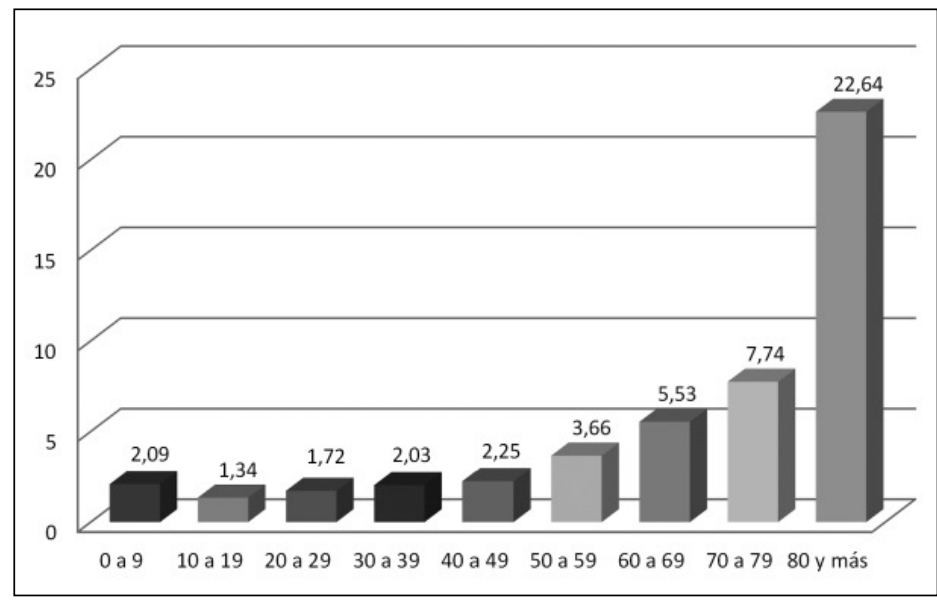

Figura 3. Tasa de fallecidos por rango de edad (por 100.000 habitantes).

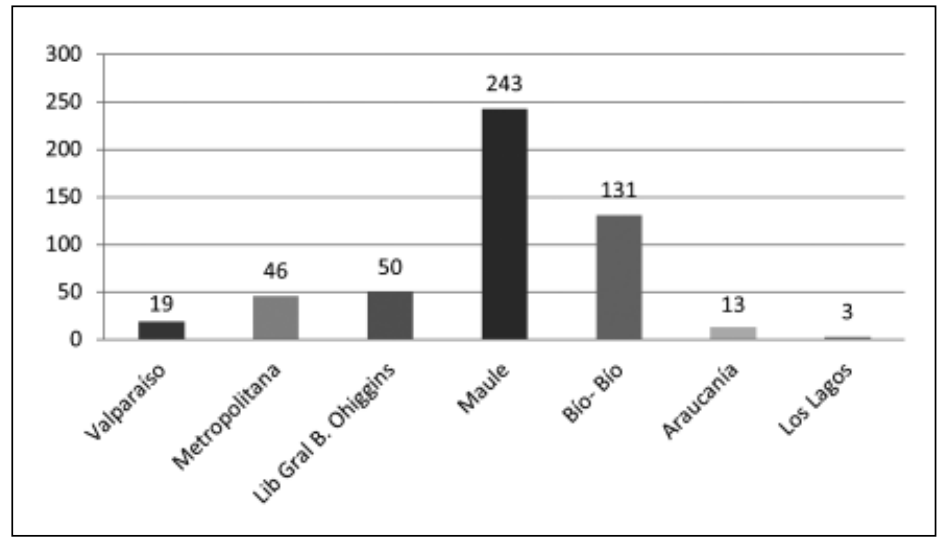

Figura 4. Número de fallecidos por Región.

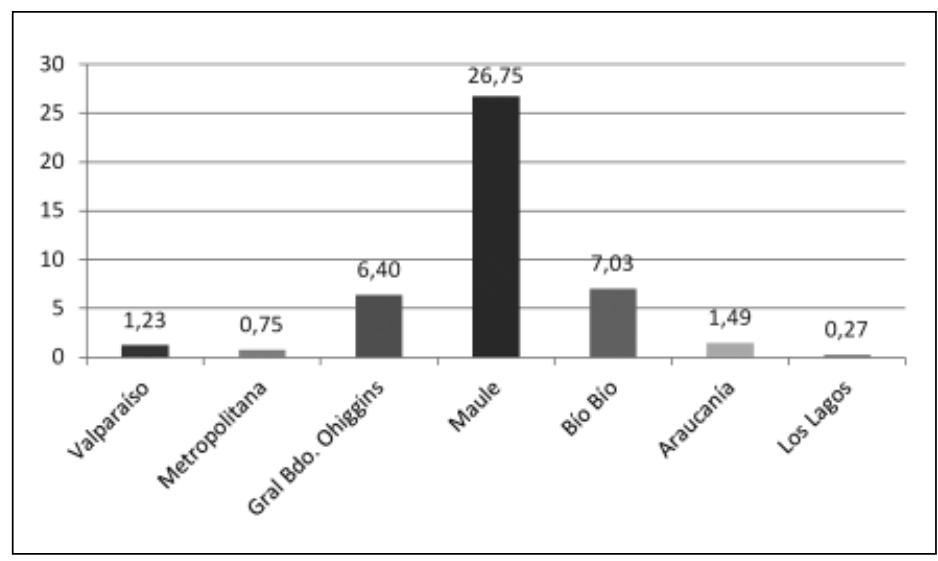

Figura 5. Tasa de fallecidos por Región (por 100.000 habitantes). 


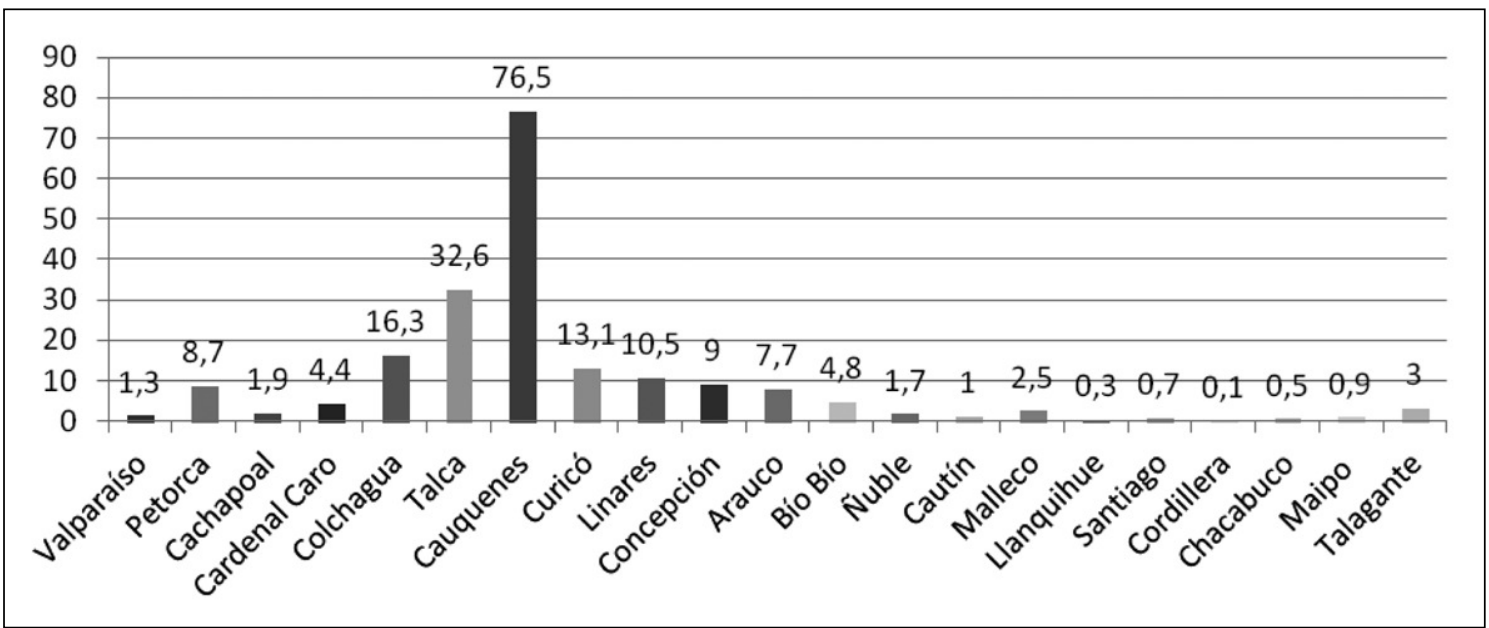

Figura 6. Tasa de fallecidos por Provincia (por 100.000 habitantes).

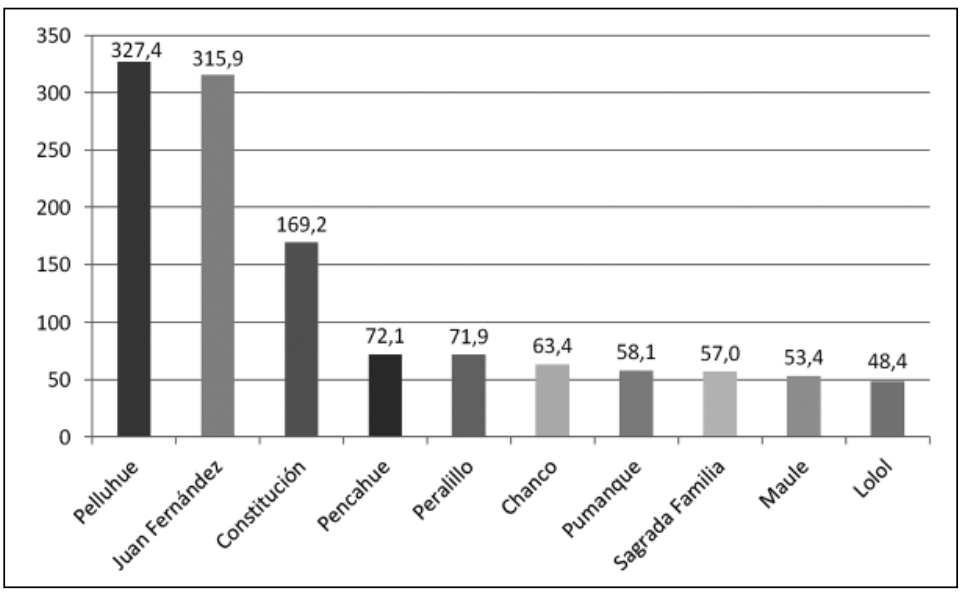

Figura 7. Comunas con mayor tasa de fallecidos (por 100.000 habitantes).

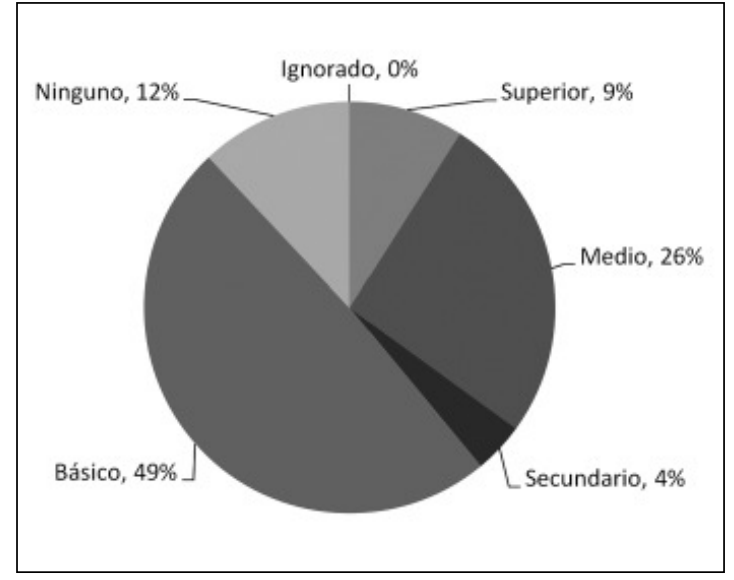

Figura 8. Distribución de la población mayor de 18 años según nivel de instrucción alcanzado.

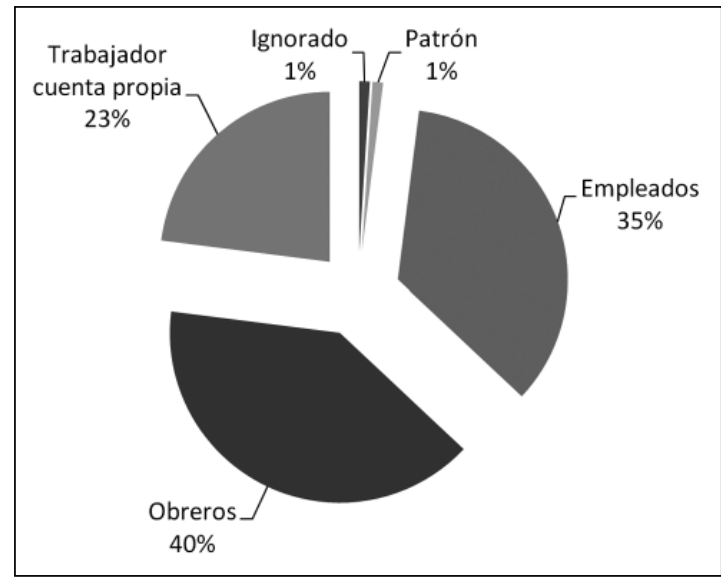

Figura 9. Población ocupada según tipo de ocupación. 
matismos, el predominio fue compromisos múltiples y secundariamente de tórax (Figuras 10 y 11).

Al comparar los tipos de traumatismos según sexo, como se observa en la Figura 12, existe una diferencia entre ambos, predominando en los hombres el traumatismo múltiple. Esta diferencia resulta significativa al aplicar la prueba de $\chi^{2}$, como prueba de asociación ${ }^{6,7}$.

La distribución de fallecidos por ahogamiento según sexo, también revela la misma diferencia, siendo mayor el número de hombres que mujeres por esta causa (Figura 13).
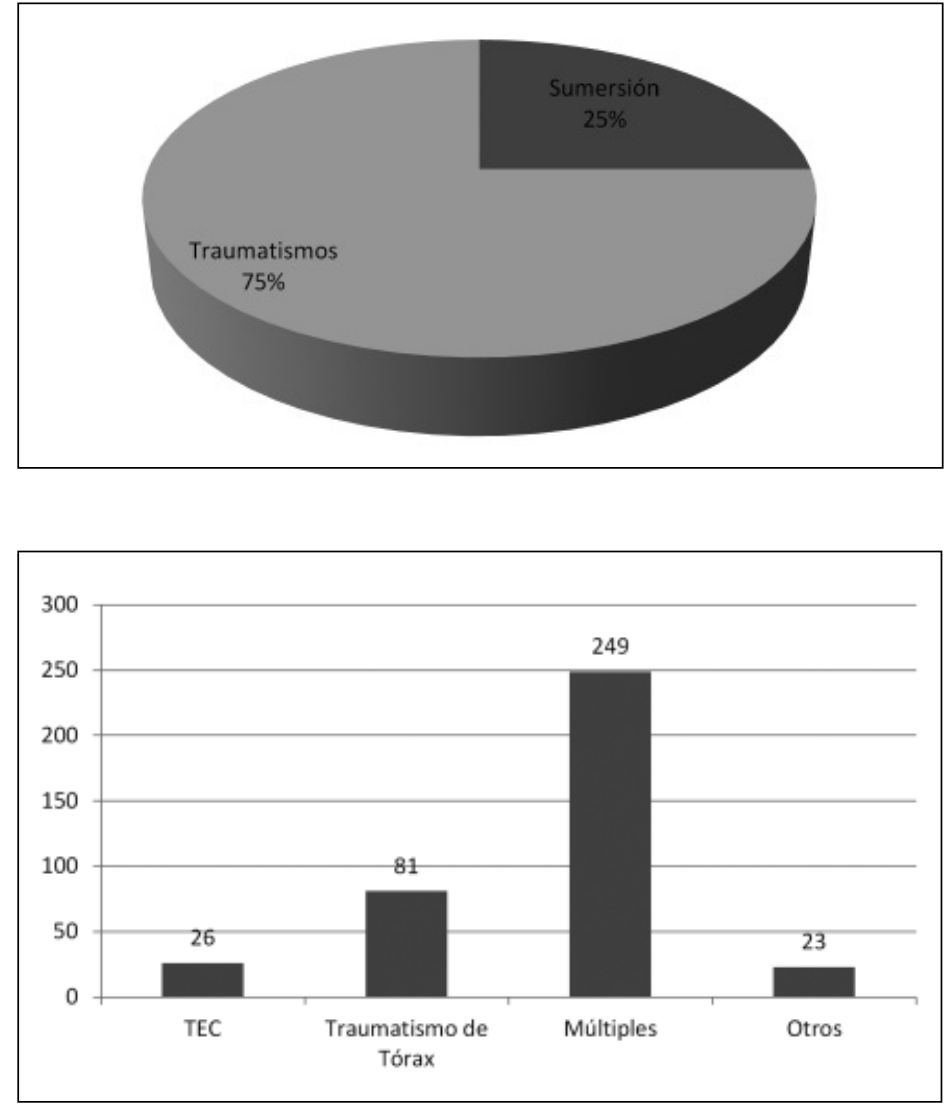

Figura 10. Diagnóstico causa básica de defunción.

Figura 11. Número de fallecidos por tipo de traumatismo.

Figura 12. Fallecidos hombres y mujeres según tipo de traumatismo. 


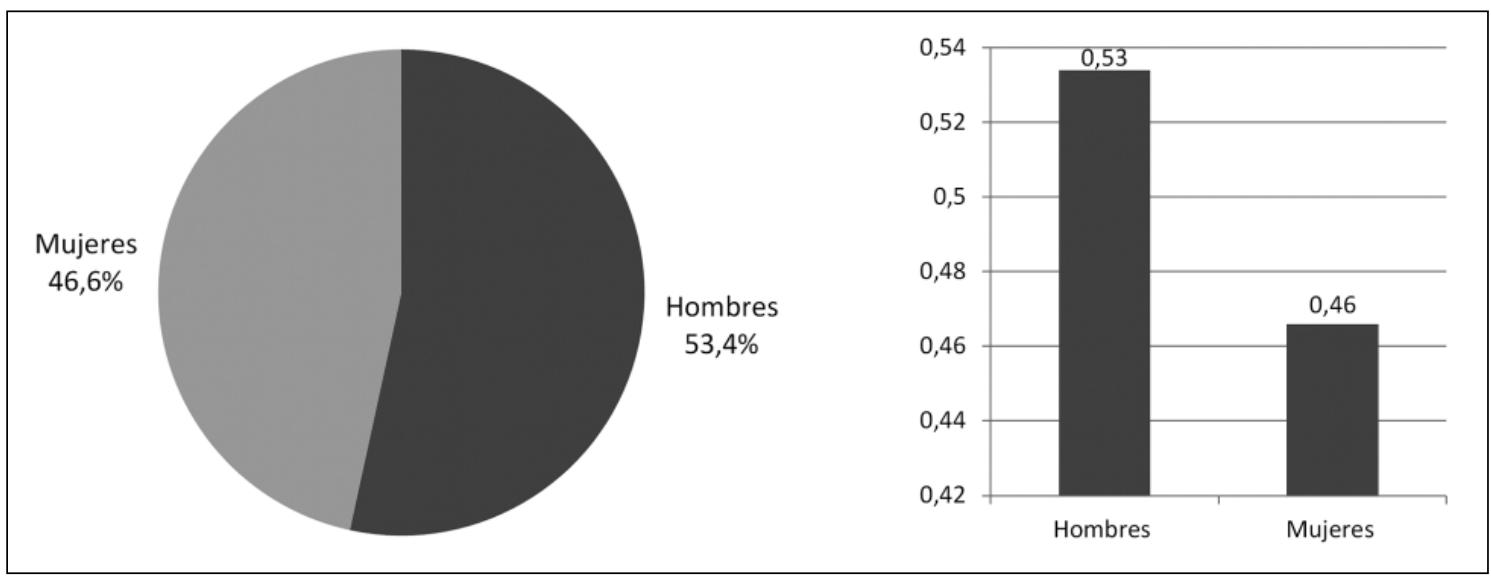

Figura 13. Distribución de fallecidos por sumersión según sexo.

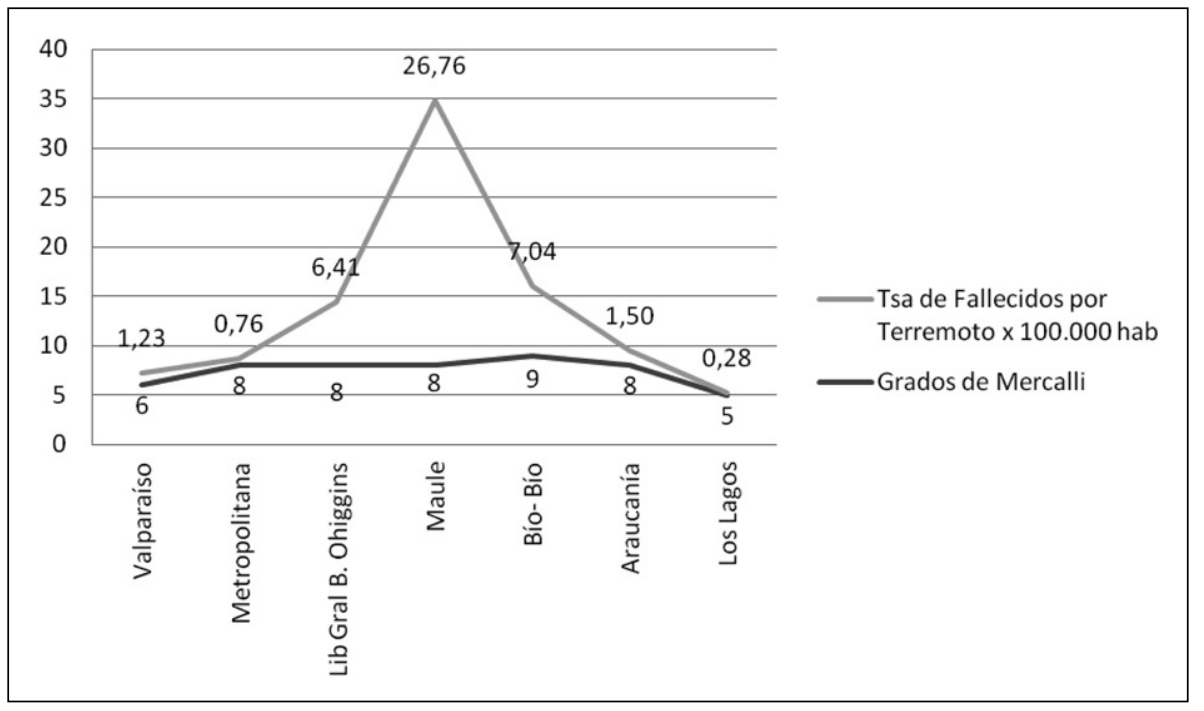

Figura 14. Tasa de fallecidos y grados de Mercalli por Región.

Finalmente, en la Figura 14, se muestra la ausencia de correlación entre el número de fallecidos por Región y la intensidad del terremoto, en grados Mercali. Esto mismo se puede corroborar en la medida del coeficiente de correlación entre esas dos variables, que muestra una débil asociación, con un resultado de 0,364.

\section{Discusión}

Como se comenta en la introducción el tema de los desastres debe constituir una preocupación en Salud Pública, en la medida que como señalan diferentes autores, si bien estos no aumentan, está provocando mayores impactos en la medida que afectan zonas cada vez más pobladas, por lo que sus impactos se prolongan más allá del cataclismo mismo ${ }^{1}$.

El terremoto y maremoto producidos en nuestro país, provocaron una cifra de fallecidos, comparativamente menor a la que eventos de intensidad similar o menor han provocado, por ejemplo en comparación con el reciente terremoto de Haití.

$\mathrm{Al}$ revisar los antecedentes clínicos y los lugares donde hubo más afectados se evidencia que los principales fallecidos son de localidades de la Región del Maule, principalmente en Talca, Cau- 
Fallecidos terremoto y maremoto Chile 2010 - J. Lastra et al

quenes, Constitución y Pelluhue y en ellos predominan los distintos síndromes de aplastamiento por terremoto y en un porcentaje menor las casos de muerte por sumersión.

Respecto del análisis de los resultados relacionados, con las variables demográficas, sociales y económicas, se identifica como principalmente afectada la población más vieja, inactivos, con bajo nivel de instrucción y ocupados en bajo nivel de jerarquía. En resumen, se puede asumir que la población más pobre, al igual que en otras condiciones, es la que presenta mayores riesgos de morir $^{8}$. Esta condición se puede explicar, de acuerdo con los estudios sobre determinantes sociales, que señalan que una baja jerarquía social, en términos de indicadores como la categoría de la ocupación y el nivel de instrucción ${ }^{9}$, constituyen factores de riesgo, condicionando estructuralmente la situación de salud y el riesgo de enfermar o morir ${ }^{10}$.

Este trabajo presentó limitaciones ya que no fue posible obtener el registro del total de fallecidos a la fecha del trabajo, faltó incorporar 10 al estudio y además se reconoce 25 posible víctimas más, que se supone fallecieron a causa del maremoto, pero cuyos cuerpos no fueron encontrados.

Finalmente, consideramos que esta materia requiere todavía mayor profundización, haciendo por ejemplo nuevos estudios, de distinto tipo, que podrían entregar mejor información de las características de los fallecidos y diseñar e implementar políticas de prevención específicas.

Agradecimientos. Agradecemos a los profesionales del Servicio Médico Legal y del Registro Civil, que de manera anónima colaboraron en la identificación de los cuerpos de los fallecidos durante el terremoto y el maremoto que azotó al país el 27 de febrero. También, a la Dirección del
Departamento de Estadística e Informaciones que consolidó la base de datos que hizo posible este estudio. También a todas las personas que trabajaron solidariamente, en distintas aéreas de trabajo, para mitigar los efectos de este tremendo desastre.

\section{Referencias}

1. OPS, Impacto de los desastres en la Salud Pública, Editor Eric K. Noji, OPS, Bogotá, 2000.

2. Naghii MR. Public health impact and medical consequences of earthquakes Rev Panam Salud Publica/Pan Am J Public Health 2005; 18 (3).

3. Sullivan KM, Hossain SM. Earthquake mortality in Pakistan, Disasters 2010; 34 (1): 176-83.

4. Chou YJ, Huang N, Lee CH, Tsai SL, Chen LS, Chang HJ. Who is at risk of death in an earthquake?; Am J Epidemiol 2004; 160 (7): 688-95.

5. Liang NJ, Shih YT, Shih FY, Wu HM, Wang HJ, Shi SF, et al. Disaster epidemiology and medical response in the Chi-Chi earthquake in Taiwan. Ann Emerg Med 2001; 38 (5): 549-55.

6. Osaki Y, Minowa M. Factors associated with earthquake deaths in the Great Hanshin-Awaji Earthquake, 1995. Nippon Koshu Eisei Zasshi 1999; 46 (3): 176-83.

7. Fiscalía Nacional (31 de enero de 2011). Nómina de Fallecidos por el tsunami del 27.02.10.

8. Evans RG, Morris L, Marmor T. Producir salud, consumir asistencia sanitaria”. En: ¿Porqué alguna gente está sana y otra no? Madrid, Editorial Díaz de Santos 1996; Pág 29-70.

9. Benach J, Muntaner C. Donde están las Causas, En: Aprender a mirar la Salud. ¿Cómo la desigualdad daña la salud nuestra Salud; Benach, J. y Muntaner, C. editores; Editorial Viejo Topo; España, 2005.

10. OMS. Comisión sobre determinantes sociales de la salud. Hacia un marco conceptual que permita analizar y actuar sobre los determinantes sociales de la salud. Documento de trabajo 5 de mayo de 2005. 